\title{
Dementias in Brazil: increasing burden in the 2000-2016 period. Estimates from the Global Burden of Disease Study 2016
}

\author{
Demências no Brasil: aumento da carga no período de 2000 a 2016. Estimativas do Estudo \\ Carga Global de Doenças 2016 \\ Simone Cecilia de MELO', Ana Paula Silva CHAMPS², Rúbia Ferreira GOULART'1, Deborah Carvalho MALTA³, \\ Valéria Maria de Azeredo PASSOS ${ }^{1,3}$
}

\begin{abstract}
Introduction: Dementia is a globally relevant health problem, which places a great burden on patients and their families. This study aimed to estimate the burden associated with Alzheimer's disease (AD) and other dementias in Brazil. Methods: In this descriptive study, we investigated the estimates obtained by the Global Burden of Disease study. We described the prevalence of AD and other dementias, years lived with disability (YLDs), age-standardized mortality, years of life lost (YLLS), and disability-adjusted life years (DALYs) among individuals aged 60 years or older between 2000 and 2016, with their respective 95\% uncertainty intervals (95\%UI). Results: During this period, the age-standardized prevalence of AD and other dementias per 100,000 people increased by 7.8\%, from 961.7 (95\%UI 828.3-1,117.5) to 1,036.9 (95\%UI 882.0-1,219.5), with approximately 1.5 million people living with dementia in Brazil. The incidence increased by 4.5\%. Similarly, all age-standardized rates had an upward trend (mortality: 3.1\%; YLLs: 5.8\%; YLDs: 7.9\%; and DALYs: 6.3\%). Mortality profiles increased with age in both years. Dementias were ranked fourth among the leading causes of death in people aged $\geq 70$ years in 2000, rising to second place in 2016. In 2016, it also represented the second and third leading causes of disability among older women and men, respectively. Conclusion: Population growth and aging have resulted in an increased burden of $\mathrm{AD}$ and other dementias in Brazil. Preventive and early diagnostic measures are essential to mitigate the burden associated with these diseases.
\end{abstract}

Keywords: Dementia; Alzheimer's Disease; Burden of Disease; Epidemiology.

\section{RESUMO}

Introdução: A demência é um problema de saúde globalmente relevante, com grande carga para os pacientes e suas famílias. Este estudo teve como objetivo estimar a carga associada à doença de Alzheimer (DA) e outras demências no Brasil. Métodos: Neste estudo descritivo, foram avaliadas as estimativas obtidas pelo estudo de Carga Global de Doença (Global Burden Disease). Descrevemos a prevalência de DA e outras demências, anos vividos com incapacidade (AVIs), mortalidade padronizada por idade, anos de vida perdidos por morte prematura (AVPs) e anos de vida perdidos ajustados por incapacidade (AVAls), com respectivos intervalos de incerteza de 95\% (95\% II), em idosos com 60+ anos, entre 2000 e 2016. Resultados: No período, a prevalência padronizada por idade de DA e de outros tipos de demência aumentou 7,8\%, de 961,7 (95\% II 828,3-1.117,5) para 1.036,9 (95\% II 882,0-1.219,5) por 100.000 pessoas, com aproximadamente 1,5 milhão de pessoas vivendo com demência no Brasil. Houve um aumento de $4,5 \%$ na incidência. Todas as taxas padronizadas por idade apresentaram tendência de aumento (mortalidade:3,1\%;AVPs: 5,8\%;AVIs: 7,9\%; e AVAls: 6,3\%). Os perfis de mortalidade demonstraram aumento com o envelhecimento, em ambos os anos. As demências ocuparam o quarto lugar entre as principais causas de morte em pessoas com 70+ anos em 2000, subindo para a segunda posição em 2016. Em 2016, também representou a segunda e terceira principais causas de incapacidade entre mulheres e homens mais velhos, respectivamente. Conclusões: 0 crescimento e envelhecimento populacional resultaram em aumento da carga de DA e de outras demências no Brasil. Medidas preventivas e de diagnóstico precoce são essenciais para atenuar a carga associada a essas doenças.

Palavras-chave: Demência; Doença de Alzheimer; Carga da Doença; Epidemiologia.

\footnotetext{
${ }^{1}$ Faculdade de Ciências Médicas de Minas Gerais, Belo Horizonte MG, Brazil.

${ }^{2}$ Hospital Sarah Belo Horizonte, Belo Horizonte MG, Brazil.

${ }^{3}$ Universidade Federal de Minas Gerais, Belo Horizonte MG, Brazil.

Simone Cecília de MELO (D) https://orcid.org/0000-0003-2022-0783; Ana Paula Silva CHAMPS (D) https://orcid.org/0000-0003-2557-8813; Rúbia Ferreira GOULART (ID https://orcid.org/0000-0002-1941-0398; Deborah Carvalho MALTA3 (D) https://orcid.org/0000-0002-8214-5734; Valéria Maria de Azeredo PASSOS (D) https://orcid.org/0000-0003-2829-5798

Correspondence: Valéria Maria de Azeredo Passos; E-mail: passos.v@gmail.com

Conflict of interest: There is no conflict of interest to declare.

Support: The Bill \& Melinda Gates Foundation supported the GBD-2016 study. The Brazilian Ministry of Health and the Brazilian National Health Fund (Fundo Nacional de Saúde, grant 25000192049/2014-14) supported the GBD-Brasil study.

Authors' contribution: SCM: contributed to the investigation, formal analysis and writing of the original draft. APSC: contributed to the investigation, formal analysis and review of the original draft. RFG: contributed to formal analysis and review of the original draft. DCM: contributed to the funding acquisition, conceptualization, and review of the original draft. VMAP: contributed to the conceptualization, investigation, methodology, formal analysis, supervision, and writing of the original draft. Received on January 28, 2020; Received in its final form on April 30, 2020; Accepted on May 31, 2020.
} 
Dementia is a neurological condition that results in a great burden to patients and their families ${ }^{1,2}$. By 2015, around 40 million people in the world had been living with dementia. This figure is estimated to double by 2040, with lower socioeconomic status (SES) countries as home to most dementia patients. The increasing dementia population is a great concern for health professionals and policymakers worldwide ${ }^{1,2}$.

Although the main causes of dementia - Alzheimer's disease (AD) and vascular dementia - are strongly associated with non-modifiable risk factors such as advancing age and positive family history, they are also related to preventable conditions such as atherosclerotic disease, cardiovascular disease, diabetes, and dyslipidemia ${ }^{3}$. In 2016, Brazil's leading risk factors related to the burden of chronic noncommunicable diseases were obesity, systolic hypertension, and hyperglycemia; all of them are associated with dementia risk and preventable 4

The Global Burden of Disease (GBD) study investigates the burden of diseases worldwide, using a standardized methodology that allows spatial and timely comparisons of estimates. Since 1990, this study has annually described the loss of health due to more than 300 diseases across 195 countries, facilitating comparisons between regions, countries, and states. Loss of health is expressed by "disability-adjusted life years" (DALYs) - the potential years of life lost due to death or disability - which combines information on mortality and morbidity ${ }^{5,6}$.

Estimates of dementia in Brazil varied greatly, even when only results of population-based or community-dwelling studies were taken into consideration. In some studies, the prevalence of dementia among Brazilian people aged 65 years or older was similar to that observed in other Latin American countries (around 7\%) . $^{7,8}$. Most Brazilian studies were conducted in the state of São Paulo. The prevalence rates of dementia among patients aged 60 years or older in the cities of Ribeirão Preto ${ }^{9}$, São Paulo ${ }^{10}$, and Tremembé ${ }^{11}$ were $12.5,12.9$, and $17.5 \%$, respectively. In a small city in the state of Minas Gerais, the figure for those aged 75 years and older was $27.5 \%^{12}$. Incidence studies are scarce. The incidence of dementia among random-selected older adults ( $\geq 65$ years) was around 14 per 1,000 person-years, twice as high as the estimate for $\mathrm{AD}^{13,14,15}$. A higher incidence of $\mathrm{AD}$ (14.8, 95\% confidence interval [95\%CI] 9.04-22.94) was found among community-dwelling older adults $(\geq 60$ years) from Porto Alegre ${ }^{16}$. These differences may reflect the lack of standardization concerning study design, diagnosis of dementia, age composition of the populations, and year of data collection.

Understanding the spatial distribution and temporal trend of the burden of dementia is the first step to implement preventive and therapeutic measures. Using the estimates of the GBD-2016 study, this investigation aimed to determine the burden of $\mathrm{AD}$ and other dementias in Brazil and its regions.

\section{METHODS}

This study aimed to describe the GBD-2016 estimates of the burden of $\mathrm{AD}$ and other dementias. All estimates derived from national and subnational data obtained and analyzed through the collaboration of a Brazilian network of researchers, the Brazilian Ministry of Health, and the Institute of Health Metrics and Evaluation (IHME) of the University of Washington ${ }^{17}$. In our study, we only used demographic and health data from the Brazilian population aged 60 years and older between 2000 and 2016 because they are more reliable than those collected in the earlier period of 1990-2000.

To express the diversity of the burden of disease across the five regions in the country, we presented the metrics of the two states of each region with the highest proportion of older people according to the 2010 census $\left.^{18}: 1\right)$ North Region: Pará (7.0\%) and Rondônia (6.3\%); 2) Northeast Region: Paraíba (11.9\%) and Bahia (10.3\%); 3) Southeast Region: Rio de Janeiro (13\%) and São Paulo (9.7\%); 4) South Region: Rio Grande do Sul (13.6\%) and Paraná (11.2\%); and 5) Midwest Region: Mato Grosso do Sul (9.7\%) and Goiás (9.3\%).

Although GBD estimates are annually provided, we investigated Brazilian estimates from GBD-2016, as a detailed description of methodology and a comprehensive worldwide analysis of $\mathrm{AD}$ and other dementias were performed with these estimates ${ }^{19}$. Dementia was defined based on the codes of the Diagnostic and Statistical Manual of Mental Disorders, $4^{\text {th }}$ and $5^{\text {th }}$ editions $(290,291.2,291.8,294$, and 331), and the International Classification of Diseases (F00, F01, F02, F03, G30, and G31), 8-10 ${ }^{\text {th }}$ editions ${ }^{19}$. Unlike most disease definitions used in the GBD study, the estimates of $\mathrm{AD}$ and other dementias were analyzed and modeled together, without considering specific causes ${ }^{19}$.

Demographic and vital data for mortality analysis were mainly provided by the Brazilian Institute of Geography and Statistics and the Mortality Information System (Brazilian Ministry of Health) ${ }^{17}$. Primary mortality data were adjusted first for underreporting and then for garbage codes. The methods for adjusting death underreporting were described by Foreman et al. ${ }^{20}$. Codes that should not be considered basic causes of death, including ill-defined causes (stated in Chapter XVIII), are classified as garbage codes. They are redistributed to other defined causes called target diseases. All garbage codes are redistributed to target diseases using statistical algorithms, judgments based on literature reviews, or proportional distributions. Details of the modeling and model validation were also published ${ }^{20,21}$. Besides the absolute number of deaths and standardized mortality by year, gender, and age, the metric years of life lost (YLLs) expresses the effect of premature deaths, which is obtained by multiplying the number of deaths by the years lost before the standard life expectancy in each age group. Life expectancy was determined based on the reference life table of the GBD study ${ }^{21}$. 
The number of years lost due to disability (YLDs) is calculated by multiplying the prevalence of each disease sequela by its disability weight ${ }^{6,19}$. Three sequelae and their respective disability weights derived from a systematic review of seven studies using the Clinical Dementia Rating (CDR) Scale: mild (CDR1), moderate (CDR2), or severe (CDR3) dementia $^{19}$. All data on the prevalence, incidence, and association with $\mathrm{AD}$ and other dementias included Brazilian populationbased studies s,8, $, 10,11,12,13,14,15$ to model the estimates. The data are available at http://ghdx.healthdata.org/gbd-2016/ data-input-sources.

Age-standardized rates were directly standardized by the age structure of the world population used by the GBD study. The metrics are presented with 95\% uncertainty intervals (95\%UIs). Uncertainties may stem from data sources and modeling steps, such as sample size variability, adjustments for mortality sources, parameter uncertainty in model estimation, specification of uncertainty for causes of death models, and differences in data availability by age, gender, year, and location ${ }^{6}$. To determine the UIs, all GBD metric calculations were performed 1,000 times so that the $95 \%$ UIs were set at the $25^{\text {th }}$ and $97.5^{\text {th }}$ of the estimated values of 1,000 estimates ${ }^{6,19}$. All results can be accessed at http://vizhub.healthdata. org/gbd-compare.

The GBD study was approved by the Institutional Review Board of the University of Washington. It also received approval from the Institutional Review Board of the Universidade Federal de Minas Gerais under the protocol CAAE - 62803316.7.0000.5149. We were not required to submit our research proposal to local institutional review boards because we accessed the GBD study data from public domain secondary databases. Individual patient data were not collected in this study; hence, informed consent was waived.

\section{RESULTS}

In Brazil, $\mathrm{AD}$ and other types of dementia were ranked fourth among the leading causes of death in people aged $\geq 70$ years in 2000, rising to second place in 2016 . However, they were not leading causes of death among people aged 50-69 years (Figure 1). Dementia was an important cause of disability among older adults, representing the second and third leading causes of disability among older women and men, respectively (Figure 2).

In 2016, estimates indicated that 1,691,024 (95\%UI 1,440,967-1,983,529) people were living with dementia in Brazil. Table 1 shows the age-standardized estimates for the entire country and its different states in the 20002016 period. Age-standardized incidence per 100,000 people increased by $4.5 \%$, from 168.8 (143.1-199.8) to 176.4
(147.3-210.8). The prevalence of dementia per 100,000 people in Brazil increased by $7.8 \%$, from 961.7 (95\%UI $828.3-1,117.5)$ to $1,036.9$ (95\%UI 882.0-1,219.5). The figures also increased across all states during the same period, and only small differences were identified among them. In 2000, the highest prevalence per 100,000 people was seen in Rio Grande do Sul (972.9 - 95\%UI 822.61,146.4) and the lowest in Bahia $(938.8$ - 95\%UI 794.81,114.8). Meanwhile, in 2016, the corresponding figures were found in São Paulo (1,156.5 - 95\%UI 994.2-1,329.1) and Rondônia (994.7 - 95\%UI 833.9-1,181.4).

Between 2000 and 2016, the increase in incidence and prevalence of dementia per 100,000 people led to a $7.9 \%$ rise in YLDs in Brazil, from 139.9 (95\%UI 98.6-185.3) to 150.9 (95\%UI 105.8-200.2). São Paulo had the highest YLDs rates per 100,000 people in this period $(144.3-95 \%$ UI $104.3-188.2$ in 2000 vs 167.2 - 95\%UI $118.3-220.8$ in 2016). Rio Grande do Sul was ranked second with respective YLDs rates per 100,000 people of 141.8 (95\%UI 99.0188.3) and 149.0 (95\%UI 104.7-197.7). The lowest figures were detected in Bahia (137.0 - 95\%UI 95.2-181.3 in 2000 vs 143.2 - 95\%UI 99.5-191.0 in 2016) and Pará (137.1 95\%UI $95.9-182.8$ in 2000 vs $143.6-95 \%$ UI $100.4-191.0$ in 2016) (Table 1).

During this 16-year period, the age-standardized mortality rate (per 100,000 people) increased by $3.1 \%$, from 52.5 (95\%UI 45.3-61.3) in 2000 to 54.2 (95\%UI 46.4-63.7) in 2016. The highest mortality rate per 100,000 people was found in Rio de Janeiro in 2016 (53.4 - 95\%UI 45.1-63.6) and the lowest in Pará in 2000 (50.1 - 95\%UI 42.5-59.5) (Table 1). Besides, the national age-standardized YLLs rates per 100,000 people increased by $5.8 \%$, from 491.9 (95\%UI 424.6-583.3) in 2000 to 520.4 (95\%UI 445.8-617.5) in 2016 (Table 1).

In particular, the DALYs per 100,000 people were 631.8 (95\%UI 538.4-744.3) in 2000, compared to 671.3 (95\%UI 565.6-799.7) in 2016. As expected, São Paulo presented the highest DALYs increase per 100,000 people (13.0\%), from 657.5 (95\%UI 574.6-747.6) in 2000 to 743.2 (95\%UI 628.2870.6) in 2016. In contrast, Paraná showed the lowest increase over this period (3.0\%) (Table 1).

During this time, the rate of DALYs increase among men was higher than among women. Men presented a 7.4\% increase in the burden of disease (DALYs per 100,000 people), from 623.8 (95\%UI 524.5-742.5) in 2000 to 670.0 (95\%UI $560.8-799.7)$ in 2016. As to women, the increasing rate was lower (5.6\%), from 636.8 (95\%UI 542.1-748.4) in 2000 to 672.2 (95\%UI 567.9-793.1) in 2016.

In 2016, the national mortality rate per 100,000 older people aged 60-64 years was 20.9 (95\%UI 15.0-20.9), compared to 421.9 (95\%UI 316.7-543.6) in those aged $75-79$ years and $1,747.7$ in those aged $\geq 80$ years. Subnational rates presented the same gradient (Table 2). 


\section{DISCUSSION}

This study identified a consistent increasing trend in all estimates, despite the confluence between UIs. This reality should not be neglected by health managers.
In 2016, dementia was the fifth leading cause of death and the $23^{\text {rd }}$ leading cause of DALYs worldwide ${ }^{19}$. Between 1990 and 2016, the prevalence of $\mathrm{AD}$ and other dementias doubled globally, from 20.2 million (95\%UI 17.4-23.5 million) to 43.8 million (95\%UI $37.8-51.0)^{18}$. The proportion
Brazil 2000 rank

Males, $50-69$ years, Deaths per 100,000

\begin{tabular}{|l|l|}
\hline 1 Ischemic heart disease & 1 Ischemic heart disease \\
\hline 2 Ischemic stroke & 2 Diabetes \\
\hline 3 COPD & 3 Intracerebral hem \\
\hline 4 Intracerebral hem & 4 Ischemic stroke \\
\hline 5 Lung cancer & 5 Lower respiratory infect \\
\hline 6 Diabetes & 6 COPD \\
\hline 7 Lower respiratory infect & 7 Lung cancer \\
\hline 8 Stomach cancer & 8 Stomach cancer \\
\hline 9 Cirrhosis hepatitis C & 9 Cirrhosis hepatitis C \\
\hline 10 Cirrhosis alcohol & 10 Cirrhosis alcohol \\
\hline 11 Esophageal cancer & 11 Esophageal cancer \\
\hline 12 Prostate cancer & 12 Prostate cancer \\
\hline 13 Pedestrian road inj & 13 Colorectal cancer \\
\hline 14 Hypertensive heart disease & 14 Alcohol use disorders \\
\hline 15 Violence firearm & 15 Other cardiomyopathy \\
\hline 16 Other cardiomyopathy & 16 Hypertensive heart disease \\
\hline 17 Colorectal cancer & 17 Violence firearm \\
\hline 18 Other cardiovascular & 18 Alzheimer disease \\
\hline 19 Diabetes CKD & 19 Diabetes CKD \\
\hline 20 Alcohol use disorders & 20 Other cardiovascular \\
\hline 21 Drug-susceptible TB & 21 Pedestrian road inj \\
\hline 22 Chagas disease & 22 Falls \\
\hline 23 Falls & 23 Motor vehicle road inj \\
\hline 24 Alzheimer disease & 24 Pancreatic cancer \\
\hline 29 Pancreatic cancer & 40 Chagas disease \\
\hline
\end{tabular}

Brazil

Females, $50-69$ years, Deaths per 100,000 2000 rank

\begin{tabular}{|c|c|}
\hline & \\
\hline 1 Ischemic heart disease & 1 Ischemic heart disease \\
\hline 2 Diabetes & 2 Diabetes \\
\hline 3 Intracerebral hem & 3 Breast cancer \\
\hline 4 Ischemic stroke & 4 Intracerebral hem \\
\hline 5 COPD & 5 COPD \\
\hline 6 Breast cancer & 6 Lung cancer \\
\hline 7 Lower respiratory infect & 7 Lower respiratory infect \\
\hline 8 Cervical cancer & 8 Ischemic stroke \\
\hline 9 Lung cancer & 9 Cervical cancer \\
\hline 10 Hypertensive heart disease & 10 Colorectal cancer \\
\hline 11 Colorectal cancer & 11 Alzheimer disease \\
\hline 12 Alzheimer disease & 12 Hypertensive heart disease \\
\hline 13 Stomach cancer & 13 Other cardiovascular \\
\hline 14 Other cardiomyopathy & 14 Stomach cancer \\
\hline 15 Other cardiovascular & 15 Pancreatic cancer \\
\hline 16 Ovarian cancer & 16 Ovarian cancer \\
\hline 17 Diabetes CKD & 17 Other neoplasms \\
\hline 18 Pancreatic cancer & 18 Diabetes CKD \\
\hline 19 Other neoplasms & 19 Other cardiomyopathy \\
\hline 20 Cirrhosis hepatitis C & 20 Brain cancer \\
\hline 21 Gallbladder cancer & 21 Cirrhosis hepatitis C \\
\hline 22 Chagas disease & 22 Urinary tract infect \\
\hline 23 Glomerulonephritis CKD & 23 Glomerulonephritis CKD \\
\hline 25 Brain cancer & 25 Gallbladder cancer \\
\hline 35 Urinary tract infect & 35 Chagas disease \\
\hline
\end{tabular}
@. $\mathrm{H} M E$ Brazil
Males, $70+$ years, Deaths per 100,000 2000 rank

\begin{tabular}{|l|l|}
\hline \multicolumn{1}{|c|}{2016 rank } \\
\hline 1 Ischemic heart disease & 1 Ischemic heart disease \\
\hline 2 COPD & 2 Alzheimer disease \\
\hline 3 Ischemic stroke & 3 COPD \\
\hline 4 Alzheimer disease & 4 Ischemic stroke \\
\hline 5 Lower respiratory infect & 5 Lower respiratory infect \\
\hline 6 Prostate cancer & 6 Prostate cancer \\
\hline 7 Diabetes & 7 Diabetes \\
\hline 8 Intracerebral hem & 8 Lung cancer \\
\hline 9 Lung cancer & 9 Intracerebral hem \\
\hline 10 Stomach cancer & 10 Hypertensive heart disease \\
\hline 11 Hypertensive heart disease & 11 Stomach cancer \\
\hline 12 Other cardiomyopathy & 12 Colorectal cancer \\
\hline 13 Other cardiovascular & 13 Other cardiovascular \\
\hline 14 Colorectal cancer & 14 Urinary tract infect \\
\hline 15 Protein-energy malnutrition & 15 Other cardiomyopathy \\
\hline 16 Aortic aneurysm & 16 Falls \\
\hline 17 Falls & 17 Diabetes CKD \\
\hline 18 Diabetes CKD & 18 Aortic aneurysm \\
\hline 19 Esophageal cancer & 19 Esophageal cancer \\
\hline 20 Pedestrian road inj & 20 Protein-energy malnutrition \\
\hline 21 Cirrhosis alcohol & 21 Hypertensive CKD \\
\hline 22 Urinary tract infect & 22 Pancreatic cancer \\
\hline 23 Cirrhosis hepatitis C & 23 Other neoplasms \\
\hline 24 Pancreatic cancer & 31 Pedestrian road inj \\
\hline 28 Other neoplasms & \\
\hline
\end{tabular}

Brazil

Females, $70+$ years, Deaths per 100,000 2000 rank

\begin{tabular}{|l|}
\hline 1 Ischemic heart disease \\
\hline 2 Ischemic stroke \\
\hline 3 Alzheimer disease \\
\hline 4 COPD \\
\hline 5 Diabetes \\
\hline 6 Lower respiratory infect \\
\hline 7 Hypertensive heart disease \\
\hline 8 Intracerebral hem \\
\hline 9 Other cardiovascular \\
\hline 10 Other cardiomyopathy \\
\hline 11 Breast cancer \\
\hline 12 Colorectal cancer \\
\hline 13 Lung cancer \\
\hline 14 Stomach cancer \\
\hline 15 Cervical cancer \\
\hline 16 Falls \\
\hline 17 Protein-energy malnutrition \\
\hline 18 Pancreatic cancer \\
\hline 19 Urinary tract infect \\
\hline 20 Atrial fibrillation \\
\hline 21 Other neoplasms \\
\hline 22 Diarrheal diseases \\
\hline 23 Diabetes CKD \\
\hline 24 Gallbladder cancer \\
\hline 25 Hypertensive CKD \\
\hline 28 Aortic aneurysm \\
\hline 1.9 \\
\hline
\end{tabular}

2016 rank

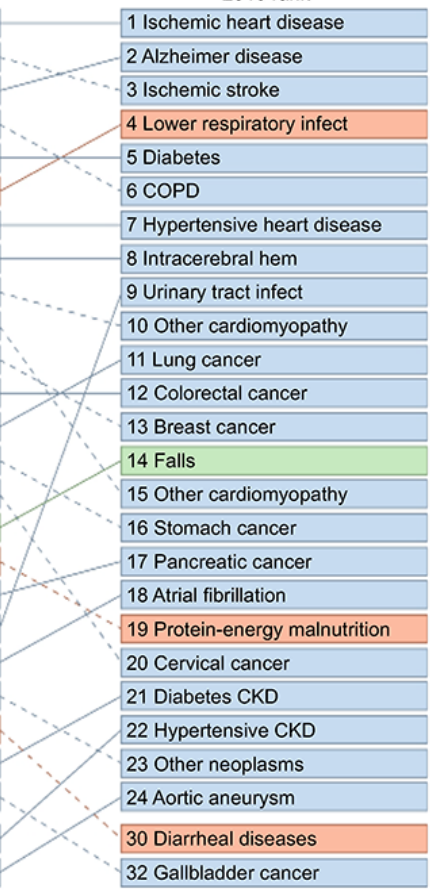

Non-communicable diseases Injurles

Figure 1. Leading causes of death among the middle-aged and older Brazilian population, from 2000 to 2016. 
of older people from a younger age group (age 65 to 69) in Latin American countries, including Brazil, was higher than in developed countries ${ }^{4}$. Besides, developed countries have more resources and social structures to face the challenges of this demographic shift; therefore, a greater impact of $\mathrm{AD}$ and other dementias could be predicted in Latin American countries.

Dementia mortality profiles by age were very similar between 2000 and 2016, but presented an important increasing gradient with age. In addition, we detected a higher
Brazil
Males, $50-69$ years, YLDs per 100,000 2000 rank

\begin{tabular}{|l|}
\hline 1 Low back pain \\
\hline 2 Age-related hearing loss \\
\hline 3 Diabetes \\
\hline 4 Other musculoskeletal \\
\hline 5 Falls \\
\hline 6 Neck pain \\
\hline 7 Major depression \\
\hline 8 Osteoarthritis \\
\hline 9 Migraine \\
\hline 10 Anxiety disorders \\
\hline 11 Edentulism \\
\hline 12 Refraction \& accommodation \\
\hline 13 Alcohol use disorders \\
\hline 14 Ischemic heart disease \\
\hline 15 COPD \\
\hline 16 Other mental \& substance \\
\hline 17 Schizophrenia \\
\hline 18 Ischemic stroke \\
\hline 19 Bipolar disorder \\
\hline 20 Periodontal diseases \\
\hline 21 Other sense organ \\
\hline 22 Dysthymia \\
\hline 23 BPH \\
\hline 24 Diarrheal diseases \\
\hline 25 Atrial fibrillation \\
\hline 26 Hemia \\
\hline
\end{tabular}

\begin{tabular}{|c|}
\hline 1 Low back pain \\
\hline 2 Age-related hearing loss \\
\hline 3 Falls \\
\hline 4 Other musculoskeletal \\
\hline 5 Diabetes \\
\hline 6 Neck pain \\
\hline 7 Osteoarthritis \\
\hline 8 Migraine \\
\hline 9 Major depression \\
\hline 10 Edentulism \\
\hline 11 Anxiety disorders \\
\hline 12 Refraction \& accommodation \\
\hline 13 Alcohol use disorders \\
\hline 14 Ischemic heart disease \\
\hline 15 Other mental \& substance \\
\hline 16 Schizophrenia \\
\hline 17 Bipolar disorder \\
\hline 18 COPD \\
\hline 19 Periodontal diseases \\
\hline 20 Other sense organ \\
\hline 21 Hemia \\
\hline 22 Ischemic stroke \\
\hline 23 Dysthymia \\
\hline 24 Atrial fibrillation \\
\hline $26 \mathrm{BPH}$ \\
\hline 30 Diarrheal diseases \\
\hline
\end{tabular}

Brazil

Females, $50-69$ years, YLDs per 100,000 2000 rank

\begin{tabular}{|c|c|}
\hline $2000 \mathrm{rank}$ & $2010 \mathrm{rank}$ \\
\hline 1 Low back pain & 1 Low back pain \\
\hline 2 Major depression & 2 Migraine \\
\hline 3 Migraine & 3 Major depression \\
\hline 4 Anxiety disorders & 4 Neck pain \\
\hline 5 Age-related hearing loss & 5 Anxiety disorders \\
\hline 6 Neck pain & 6 Age-related hearing loss \\
\hline 7 Diabetes & 7 Other musculoskeletal \\
\hline 8 Other musculoskeletal & 8 Diabetes \\
\hline 9 Edentulism & 9 Osteoarthritis \\
\hline 10 Osteoarthritis & 10 Edentulism \\
\hline 11 Refraction \& accommodation & 11 Refraction \& accommodation \\
\hline 12 Falls & 12 Falls \\
\hline 13 Dietary iron deficiency & 13 Dietary iron deficiency \\
\hline 14 Bipolar disorder & 14 Bipolar disorder \\
\hline 15 Rheumatoid arthritis & 15 Rheumatoid arthritis \\
\hline 16 Dysthymia & 16 Dysthymia \\
\hline 17 Other sense organ & 17 Other sense organ \\
\hline 18 Tension headache & 18 Schizophrenia \\
\hline 19 Schizophrenia & 19 Tension headache \\
\hline 20 Ischemic stroke & 20 Other mental \& substance \\
\hline 21 Diarrheal diseases & 21 Ischemic heart disease \\
\hline 22 Other mental \& substance & 22 COPD \\
\hline 23 COPD & 23 Periodontal diseases \\
\hline 24 Ischemic heart disease & 24 Ischemic stroke \\
\hline 25 Periodontal diseases & 25 Diarrheal diseases \\
\hline
\end{tabular}
IHME
Brazil Males, 70+ years, YLDs per 100,000

\begin{tabular}{|c|c|}
\hline \multicolumn{2}{|r|}{ per } \\
\hline 2000 rank & 2016 rank \\
\hline 1 Age-related hearing loss & $1 \mathrm{Age}$-related hearing loss \\
\hline 2 Low back pain & 2 Alzheimer disease \\
\hline 3 Alzheimer disease & 3 Low back pain \\
\hline 4 Edentulism & 4 Falls \\
\hline 5 Falls & 5 Diabetes \\
\hline 6 Diabetes & 6 Edentulism \\
\hline 7 Osteoarthritis & 7 Osteoarthritis \\
\hline 8 Ischemic heart disease & 8 Ischemic heart disease \\
\hline 9 Ischemic stroke & 9 Refraction \& accommodation \\
\hline 10 Other musculoskeletal & 10 Ischemic stroke \\
\hline 11 Refraction \& accommodation & 11 Neck pain \\
\hline 12 Neck pain & 12 Cataract \\
\hline 13 Major depression & 13 Atrial fibrillation \\
\hline 14 Atrial fibrillation & 14 Major depression \\
\hline 15 Cataract & 15 COPD \\
\hline 16 COPD & 16 Anxiety disorders \\
\hline 17 Diarrheal diseases & 17 Other sense organ \\
\hline 18 Anxiety disorders & 18 Diarrheal diseases \\
\hline $19 \mathrm{BPH}$ & $19 \mathrm{BPH}$ \\
\hline 20 Other sense organ & 20 Migraine \\
\hline 21 Migraine & 21 Other cardiovascular \\
\hline 22 Other cardiovascular & 22 Prostate cancer \\
\hline 23 Prostate cancer & 23 Other unintentional \\
\hline 24 Other unintentional & 24 Other musculoskeletal \\
\hline 25 Diabetes CKD & 25 Hernia \\
\hline 28 Hernia & 29 Diabetes CKD \\
\hline
\end{tabular}

Brazil

Females, $70+$ years, YLDs per 100,000 2000 rank 2016 rank

\begin{tabular}{|c|c|}
\hline & \\
\hline 1 Age-related hearing loss & -1 Age-related hearing loss \\
\hline 2 Low back pain & 2 Low back pain \\
\hline 3 Alzheimer disease & 3 Alzheimer disease \\
\hline 4 Edentulism & 4 Edentulism \\
\hline 5 Diabetes & 5 Falls \\
\hline 6 Major depression & 6 Diabetes \\
\hline 7 Osteoarthritis & 7 Osteoarthritis \\
\hline 8 Falls & 8 Major depression \\
\hline 9 Neck pain & 9 Neck pain \\
\hline 10 Refraction \& accommodation & 10 Refraction \& accommodation \\
\hline 11 Ischemic stroke & 11 Cataract \\
\hline 12 Cataract & 12 Ischemic stroke \\
\hline 13 Dietary iron deficiency & 13 Dietary iron deficiency \\
\hline 14 Other musculoskeletal & 14 Anxiety disorders \\
\hline 15 Anxiety disorders & 15 Other musculoskeletal \\
\hline 16 Migraine & 16 Migraine \\
\hline 17 Ischemic heart disease & 17 Ischemic heart disease \\
\hline 18 Atrial fibrillation & 18 Atrial fibrillation \\
\hline 19 Other sense organ & 19 Other sense organ \\
\hline 20 Diarrheal diseases & 20 Diarrheal diseases \\
\hline 21 Rheumatoid arthritis & 21 Rheumatoid arthritis \\
\hline 22 COPD & 22 COPD \\
\hline 23 Other cardiovascular & 23 Other cardiovascular \\
\hline 24 Asthma & 24 Dysthymia \\
\hline 25 Dysthymia & 25 Fungal skin diseases \\
\hline 28.Fungal skin diseases & 31 Asthma \\
\hline
\end{tabular}
28. Fungal skin

\footnotetext{
Non-communicable diseases Injurles
}

Figure 2. Leading causes of disability among the middle-aged and older Brazilian population, from 2000 to 2016. 
increase in the age-standardized prevalence than in the incidence of $\mathrm{AD}$ and other dementias. This finding probably results from population growth and aging rather than an increase in the risk of disease in this period.

Aging represents a victory for humanity; however, it may also represent a greater risk of chronic diseases and disability. This situation may compromise the autonomy of older people. Brazil has been experiencing a rapid and marked aging process. Its older population in 1940 consisted of 1.7 million people (4\%), increasing to 14.5 million $(8.6 \%)$ in 2000 . The aging rate is higher among those aged 80 years or older and shows female predominance $(55 \%)^{22}$. As a result, in 2016, Brazil had the second highest age-standardized prevalence of $\mathrm{AD}$ and other dementias per 100,000 people $(1,037-95 \%$ UI $882-1,220)$, after only Turkey $(1,192-95 \% \text { UI } 1,007-1,405)^{19}$.

The burden of $\mathrm{AD}$ and other dementias increased by $6.3 \%$ in Brazil. Chronic degenerative diseases such as dementia are associated with higher morbidity, prevalence, and YLDs than mortality and YLLs. Increased longevity implies that the gain in life expectancy is followed by a greater degree of health $\operatorname{loss}^{22,23}$. Prevalence increase will become a reality, as most dementias are progressive in nature and have no treatment to modify their course.

To face the burden profile of dementia, health financing must increase pari passu with population growth and aging. A recent study showed that the overall cost of dementia in Brazil (\$1,405.72 per capita) is significantly

Table 1. Age-standardized incidence, prevalence, mortality, years lived with disability, years of life lost, and disability-adjusted life year of Alzheimer's disease and other dementias in Brazil from 2000 to 2016.

\begin{tabular}{|c|c|c|c|c|c|c|c|c|c|c|c|c|c|c|c|c|c|c|}
\hline \multirow{2}{*}{ Location } & \multicolumn{3}{|c|}{$\begin{array}{c}\text { Incidence (95\%UI) } \\
\times 100,000 \text { inhabitants }\end{array}$} & \multicolumn{3}{|c|}{$\begin{array}{c}\text { Prevalence }(95 \% \text { UI) } \\
\times 100,000 \text { inhabitants }\end{array}$} & \multicolumn{3}{|c|}{$\begin{array}{c}\text { Mortality (95\%UI) } \\
\times 100,000 \text { inhabitants }\end{array}$} & \multicolumn{3}{|c|}{$\begin{array}{c}\text { YLDs }(95 \% \text { UI) } \\
\times 100,000 \text { inhabitants }\end{array}$} & \multicolumn{3}{|c|}{$\begin{array}{c}\text { YLLs (95\%UI) } \\
\times 100,000 \text { inhabitants }\end{array}$} & \multicolumn{3}{|c|}{$\begin{array}{c}\text { DALYs (95\%UI) } \\
\times 100,000 \text { inhabitants }\end{array}$} \\
\hline & 2000 & 2016 & $\Delta \%$ & 2000 & 2016 & $\Delta \%$ & 2000 & 2016 & $\Delta \%$ & 2000 & 2016 & $\Delta \%$ & 2000 & 2016 & $\Delta \%$ & 2000 & 2016 & $\Delta \%$ \\
\hline Brazil & $\begin{array}{l}168.8 \\
143.1- \\
199.8\end{array}$ & $\begin{array}{l}176.4 \\
147.3- \\
210.8\end{array}$ & 4.5 & $\begin{array}{l}961.7 \\
828.3- \\
1117.5\end{array}$ & $\begin{array}{l}1036.9 \\
882.0- \\
1219.5\end{array}$ & 7.8 & $\begin{array}{l}52.5 \\
45.3- \\
61.6\end{array}$ & $\begin{array}{l}54.2 \\
46.4- \\
63.7\end{array}$ & 3.1 & $\begin{array}{l}139.9 \\
98.6- \\
185.3\end{array}$ & $\begin{array}{c}150.9 \\
105.8- \\
200.2\end{array}$ & 7.9 & $\begin{array}{l}491.9 \\
424.6- \\
583.3\end{array}$ & $\begin{array}{c}520.4 \\
445.8- \\
617.5\end{array}$ & 5.8 & $\begin{array}{c}631.8 \\
538.4- \\
744.3\end{array}$ & $\begin{array}{c}671.3 \\
565.6- \\
799.7\end{array}$ & 6.3 \\
\hline Men & $\begin{array}{c}159.5 \\
135.2- \\
188.1\end{array}$ & $\begin{array}{l}169.9 \\
142.1- \\
204.7\end{array}$ & 6.5 & $\begin{array}{l}909.0 \\
780.2- \\
1061.0\end{array}$ & $\begin{array}{l}995.6 \\
846.6- \\
1175.3\end{array}$ & 9.5 & $\begin{array}{l}52.2 \\
44.3- \\
61.8\end{array}$ & $\begin{array}{l}54.3 \\
46.2- \\
64.4\end{array}$ & 4.1 & $\begin{array}{l}131.9 \\
92.9- \\
175.1\end{array}$ & $\begin{array}{c}144.6 \\
101.0- \\
192.0\end{array}$ & 9.6 & $\begin{array}{l}491.9 \\
420.8- \\
586.4\end{array}$ & $\begin{array}{c}525.4 \\
449.6- \\
627.0\end{array}$ & 6.8 & $\begin{array}{l}623.8 \\
524.5- \\
742.5\end{array}$ & $\begin{array}{l}670.0 \\
560.8^{-} \\
799.7\end{array}$ & 7.4 \\
\hline Women & $\begin{array}{c}174.5 \\
148.0- \\
206.7\end{array}$ & $\begin{array}{c}180.6 \\
150.8- \\
214.9\end{array}$ & 3.5 & $\begin{array}{l}993.2 \\
857.5- \\
1156.3\end{array}$ & $\begin{array}{l}1064.2 \\
905.0- \\
1245.3\end{array}$ & 7.1 & $\begin{array}{c}52.7 \\
45.4- \\
61.4\end{array}$ & $\begin{array}{c}54.1 \\
46.5- \\
63.1\end{array}$ & 2.5 & $\begin{array}{l}144.5 \\
102.1- \\
190.6\end{array}$ & $\begin{array}{l}154.9 \\
108.1- \\
204.8\end{array}$ & 7.2 & $\begin{array}{l}492.2 \\
425.7- \\
579.2\end{array}$ & $\begin{array}{c}517.3 \\
441.9- \\
611.8\end{array}$ & 5.1 & $\begin{array}{l}636.8 \\
542.1- \\
748.4\end{array}$ & $\begin{array}{l}672.2 \\
567.9- \\
793.1\end{array}$ & 5.6 \\
\hline \multicolumn{19}{|l|}{ Midwest } \\
\hline Goiás & $\begin{array}{c}165.6 \\
138.4- \\
199.7\end{array}$ & $\begin{array}{c}168.7 \\
140.8- \\
203.8\end{array}$ & 1.9 & $\begin{array}{l}944.5 \\
797.7- \\
1116.8\end{array}$ & $\begin{array}{c}989.2 \\
829.3- \\
1174.7\end{array}$ & 4.7 & $\begin{array}{c}52.2 \\
43.5- \\
62.3\end{array}$ & $\begin{array}{c}53.2 \\
44.2- \\
63.5\end{array}$ & 1.8 & $\begin{array}{l}137.5 \\
96.0- \\
182.3\end{array}$ & $\begin{array}{c}143.7 \\
100.3- \\
191.5\end{array}$ & 4.5 & $\begin{array}{l}487.1 \\
408.7- \\
586.3\end{array}$ & $\begin{array}{l}508.0 \\
420.1- \\
612.1\end{array}$ & 4.3 & $\begin{array}{l}624.7 \\
520.0- \\
747.8\end{array}$ & $\begin{array}{c}651.8 \\
538.5- \\
789.7\end{array}$ & 4.3 \\
\hline $\begin{array}{l}\text { Mato } \\
\text { Grosso } \\
\text { do Sul }\end{array}$ & $\begin{array}{c}166.9 \\
139.1- \\
201.4\end{array}$ & $\begin{array}{c}170.5 \\
141.3- \\
207.3\end{array}$ & 2.2 & $\begin{array}{l}952.0 \\
806.3- \\
1125.0\end{array}$ & $\begin{array}{c}998.8 \\
839.8- \\
1180.3\end{array}$ & 4.9 & $\begin{array}{l}53.4 \\
44.3- \\
64.0\end{array}$ & $\begin{array}{l}53.8 \\
44.9- \\
64.5\end{array}$ & 0.8 & $\begin{array}{l}138.7 \\
97.2- \\
184.1\end{array}$ & $\begin{array}{c}145.3 \\
101.6- \\
192.9\end{array}$ & 4.8 & $\begin{array}{l}500.6 \\
418.4- \\
605.4\end{array}$ & $\begin{array}{c}513.9 \\
424.2- \\
617.2\end{array}$ & 2.7 & $\begin{array}{c}639.2 \\
533.9- \\
768.8\end{array}$ & $\begin{array}{l}659.2 \\
541.1- \\
787.1\end{array}$ & 3.1 \\
\hline \multicolumn{19}{|l|}{ North } \\
\hline Pará & $\begin{array}{l}164.7 \\
137.1- \\
200.1\end{array}$ & $\begin{array}{l}168.1 \\
39.0- \\
202.7\end{array}$ & 2.1 & $\begin{array}{c}939.8 \\
796.0- \\
1112.7\end{array}$ & $\begin{array}{c}984.9 \\
818.6- \\
1168.9\end{array}$ & 4.8 & $\begin{array}{c}50.1 \\
42.5- \\
59.5\end{array}$ & $\begin{array}{c}51.6 \\
42.9- \\
62.3\end{array}$ & 3.1 & $\begin{array}{c}137.1 \\
95.9- \\
182.8\end{array}$ & $\begin{array}{c}143.6 \\
100.4- \\
191.0\end{array}$ & 4.8 & $\begin{array}{l}467.5 \\
394.1- \\
559.6\end{array}$ & $\begin{array}{l}494.2 \\
412.0- \\
605.9\end{array}$ & 5.7 & $\begin{array}{c}604.6 \\
503.6- \\
723.2\end{array}$ & $\begin{array}{c}637.8 \\
528.4^{-} \\
777.6\end{array}$ & 5.5 \\
\hline Rondônia & $\begin{array}{c}166.2 \\
138.8- \\
199.1\end{array}$ & $\begin{array}{c}169.9 \\
140.4- \\
206.0\end{array}$ & 2.2 & $\begin{array}{c}948.1 \\
803.0- \\
1119.0\end{array}$ & $\begin{array}{c}994.7 \\
833.9- \\
1181.4\end{array}$ & 4.9 & $\begin{array}{c}54.7 \\
45.7- \\
65.3\end{array}$ & $\begin{array}{c}55.1 \\
45.5- \\
66.3\end{array}$ & 0.8 & $\begin{array}{l}138.2 \\
95.7- \\
182.4\end{array}$ & $\begin{array}{c}145.3 \\
100.8- \\
194.1\end{array}$ & 5.2 & $\begin{array}{l}512.0 \\
430.2- \\
616.3\end{array}$ & $\begin{array}{l}527.6 \\
435.4- \\
643.2\end{array}$ & 3.0 & $\begin{array}{l}650.2 \\
543.8- \\
780.5\end{array}$ & $\begin{array}{l}672.9 \\
552.1- \\
817.4\end{array}$ & 3.5 \\
\hline \multicolumn{19}{|l|}{ Northeast } \\
\hline Paraíba & $\begin{array}{c}166.3 \\
138.8- \\
200.3\end{array}$ & $\begin{array}{c}170.0 \\
141.4- \\
205.6\end{array}$ & 2.2 & $\begin{array}{l}948.1 \\
801.2- \\
1121.1\end{array}$ & $\begin{array}{l}996.5 \\
841.6- \\
1179.4\end{array}$ & 5.1 & $\begin{array}{c}51.8 \\
43.6- \\
62.0\end{array}$ & $\begin{array}{c}52.5 \\
43.6- \\
63.1\end{array}$ & 0.5 & $\begin{array}{l}138.5 \\
97.2- \\
184.3\end{array}$ & $\begin{array}{c}145.5 \\
101.9- \\
193.0\end{array}$ & 5.0 & $\begin{array}{l}487.9 \\
411.1- \\
591.0\end{array}$ & $\begin{array}{c}502.4 \\
416.6- \\
608.0\end{array}$ & 3.0 & $\begin{array}{l}626.4 \\
526.4- \\
761.2\end{array}$ & $\begin{array}{l}647.9 \\
534.8- \\
785.1\end{array}$ & 3.4 \\
\hline Bahia & $\begin{array}{l}164.6 \\
137.0- \\
198.6\end{array}$ & $\begin{array}{c}167.9 \\
139.0- \\
204.1\end{array}$ & 2.0 & $\begin{array}{c}938.8 \\
794.8- \\
1114.8\end{array}$ & $\begin{array}{c}983.4 \\
822.2- \\
1116.4\end{array}$ & 4.7 & $\begin{array}{c}50.2 \\
42.6- \\
49.8\end{array}$ & $\begin{array}{c}50.6 \\
42.4- \\
61.1\end{array}$ & 0.8 & $\begin{array}{l}137.0 \\
95.2- \\
181.3\end{array}$ & $\begin{array}{l}143.2 \\
99.5- \\
191.0\end{array}$ & 4.6 & $\begin{array}{c}468.9 \\
393.8- \\
563.3\end{array}$ & $\begin{array}{l}485.0 \\
400.9- \\
594.9\end{array}$ & 3.4 & $\begin{array}{l}605.9 \\
510.0- \\
725.3\end{array}$ & $\begin{array}{l}628.2 \\
515.1- \\
760.6\end{array}$ & 3.7 \\
\hline \multicolumn{19}{|l|}{ South } \\
\hline Paraná & $\begin{array}{c}168.7 \\
140.2- \\
203.9\end{array}$ & $\begin{array}{c}172.1 \\
142.8- \\
208.3\end{array}$ & 2.0 & $\begin{array}{c}962.4 \\
809.8^{-} \\
1138.4\end{array}$ & $\begin{array}{l}1008.1 \\
848.9- \\
1199.0\end{array}$ & 4.8 & $\begin{array}{c}53.0 \\
44.4- \\
63.3\end{array}$ & $\begin{array}{c}53.3 \\
44.7- \\
64.2\end{array}$ & 0.5 & $\begin{array}{l}139.8 \\
96.7- \\
185.2\end{array}$ & $\begin{array}{c}146.4 \\
102.8- \\
193.9\end{array}$ & 4.7 & $\begin{array}{l}497.0 \\
415.7- \\
599.0\end{array}$ & $\begin{array}{l}509.6 \\
416.7- \\
618.3\end{array}$ & 2.5 & $\begin{array}{c}636.8 \\
530.0- \\
767.0\end{array}$ & $\begin{array}{l}655.9 \\
537.1- \\
793.3\end{array}$ & 3.0 \\
\hline $\begin{array}{l}\text { Rio } \\
\text { Grande } \\
\text { do Sul }\end{array}$ & $\begin{array}{c}170.5 \\
141.4- \\
205.4\end{array}$ & $\begin{array}{l}174.2 \\
144.7- \\
210.8\end{array}$ & 2.2 & $\begin{array}{c}972.9 \\
822.6- \\
1146.4\end{array}$ & $\begin{array}{l}1021.2 \\
862.7- \\
1205.7\end{array}$ & 5.0 & $\begin{array}{c}52.8 \\
43.9- \\
63.1\end{array}$ & $\begin{array}{c}53.3 \\
44.7- \\
63.9\end{array}$ & 0.8 & $\begin{array}{l}141.8 \\
99.0- \\
188.3\end{array}$ & $\begin{array}{c}149.0 \\
104.7- \\
197.7\end{array}$ & 5.1 & $\begin{array}{l}494.1 \\
412.2- \\
598.5\end{array}$ & $\begin{array}{l}510.3 \\
425.2- \\
619.6\end{array}$ & 3.3 & $\begin{array}{l}635.9 \\
526.5- \\
764.6\end{array}$ & $\begin{array}{c}659.3 \\
545.5- \\
787.1\end{array}$ & 3.7 \\
\hline \multicolumn{19}{|l|}{ Southeast } \\
\hline $\begin{array}{l}\text { Rio de } \\
\text { Janeiro }\end{array}$ & $\begin{array}{c}167.2 \\
139.0- \\
201.7\end{array}$ & $\begin{array}{l}170.5 \\
141.2- \\
206.5\end{array}$ & 1.9 & $\begin{array}{l}952.2 \\
802.1- \\
1129.2\end{array}$ & $\begin{array}{l}999.3 \\
839.3- \\
1192.0\end{array}$ & 4.9 & $\begin{array}{c}53.1 \\
44.0- \\
63.8\end{array}$ & $\begin{array}{l}53.4 \\
45.1- \\
63.6\end{array}$ & 0.5 & $\begin{array}{l}138.9 \\
97.5- \\
183.7\end{array}$ & $\begin{array}{c}146.1 \\
101.7- \\
193.4\end{array}$ & 5.2 & $\begin{array}{c}497.5 \\
416.0- \\
601.7\end{array}$ & $\begin{array}{c}509.7 \\
424.4- \\
619.8\end{array}$ & 2.5 & $\begin{array}{c}636.4 \\
530.3- \\
764.0\end{array}$ & $\begin{array}{c}655.8 \\
543.7- \\
791.8\end{array}$ & 3.1 \\
\hline $\begin{array}{l}\text { São } \\
\text { Paulo }\end{array}$ & $\begin{array}{c}176.5 \\
156.4- \\
200.3\end{array}$ & $\begin{array}{c}194.9 \\
166.6- \\
227.8\end{array}$ & 10.5 & $\begin{array}{c}900.1 \\
900.1- \\
1106.0\end{array}$ & $\begin{array}{l}1156.5 \\
994.2- \\
1329.1\end{array}$ & 15.8 & $\begin{array}{l}55.1 \\
47.4- \\
63.8\end{array}$ & $\begin{array}{l}59.4 \\
50.7- \\
68.9\end{array}$ & 7.7 & $\begin{array}{c}144.3 \\
104.3- \\
188.2\end{array}$ & $\begin{array}{c}167.2 \\
118.3- \\
220.8\end{array}$ & 15.9 & $\begin{array}{c}513.3 \\
446.2- \\
590.9\end{array}$ & $\begin{array}{l}575.9 \\
491.0- \\
673.8\end{array}$ & 12.2 & $\begin{array}{c}657.5 \\
574.6- \\
747.6\end{array}$ & $\begin{array}{c}743.2 \\
628.2- \\
870.6\end{array}$ & 13.0 \\
\hline
\end{tabular}

YLDs: years lived with disability; YLLs: years of life lost; DALYs: disability-adjusted life years; $95 \% U \mathrm{UI}$ : 95\% uncertainty interval; $\Delta \%$ : annual percentage change. 
Table 2. Mortality and morbidity rates of Alzheimer's disease and other dementias by age in Brazil, in 2000 and 2016.

\begin{tabular}{|c|c|c|c|c|c|c|c|c|c|c|}
\hline \multirow{2}{*}{$\begin{array}{l}\text { Age in years } \\
\text { by location } \\
\text { Brazil } \\
\end{array}$} & \multicolumn{5}{|c|}{$\begin{array}{c}\text { Mortality rates } \\
\text { (per } 100,000 \text { inhabitants) }\end{array}$} & \multicolumn{5}{|c|}{$\begin{array}{c}\text { YLDs rates } \\
\text { (per 100,000 inhabitants) }\end{array}$} \\
\hline & 2000 & $95 \%$ UI & 2016 & $95 \%$ UI & $\Delta \%$ & 2000 & $95 \% \mathrm{UI}$ & 2016 & $95 \%$ UI & $\Delta \%$ \\
\hline $60-64$ & 21.0 & $15.2-28.9$ & 20.9 & $15.0-29.3$ & -0.1 & 158.7 & $104.6-236.2$ & 159.0 & $104.1-233.8$ & 0.2 \\
\hline $65-69$ & 54.5 & $40.1-72.7$ & 59.4 & $43.3-80.0$ & 9.0 & 325.7 & $212.9-475.8$ & 357.7 & $232.2-532.2$ & 9.8 \\
\hline $70-74$ & 145.0 & $106.5-193.9$ & 167.1 & $123.5-223.8$ & 15.2 & 733.2 & $484.5-1020.6$ & 853.8 & $554.7-1199.9$ & 16.4 \\
\hline $75-79$ & 371.3 & $280.9-473.5$ & 421.9 & $316.7-543.6$ & 13.6 & 1298.2 & $869.2-1825.3$ & 1507.2 & $995.4-2157.2$ & 16.1 \\
\hline $80-84$ & 846.6 & $656.5-1090.6$ & 921.2 & 711.7-1178.9 & 8.8 & 2549.9 & $1724.6-3561.9$ & 2824.5 & $1891.9-3997.8$ & 10.8 \\
\hline $85-89$ & 1902.3 & $1496.3-2362.8$ & 1916.7 & $1508.3-2378.8$ & 0.8 & 3770.1 & $2526.1-5124.9$ & 3881.0 & $2620.0-5334.5$ & 2.9 \\
\hline $90-94$ & 4155.4 & $3340.1-4980.2$ & 3938.6 & $3193.8-4698.7$ & -5.2 & 4939.5 & $3317.6-6683.1$ & 4722.5 & $3157.4-6373.2$ & -4.4 \\
\hline $95+$ & 6303.6 & 4969.5-7876.2 & 5744.0 & $4545.2-7189.7$ & -8.9 & 5961.8 & $3913.2-8294.0$ & 5449.5 & $3563.2-7637.1$ & -8.6 \\
\hline
\end{tabular}

\section{Midwest region}

Mato Grosso do Sul

$\begin{array}{ccccccccccc}60-64 & 20.7 & 14.8-29.2 & 20.3 & 14.5-28.3 & -2.1 & 153.3 & 97.2-231.2 & 151.6 & 95.0-228.6 & -1.1 \\ 65-69 & 54.9 & 39.3-75.4 & 57.8 & 40.8-79.8 & 5.2 & 319.9 & 201.8-470.5 & 339.1 & 212.5-503.2 & 6.0 \\ 70-74 & 148.5 & 104.9-204.8 & 162.7 & 114.5-223.4 & 9.6 & 731.3 & 468.4-1042.5 & 816.7 & 540.5-1154.5 & 11.7 \\ 75-79 & 382.0 & 277.6-510.5 & 415.4 & 297.5-554.5 & 8.7 & 1298.7 & 860.4-1831.9 & 1450.6 & 947.2-2046.5 & 11.7 \\ 80-84 & 864.5 & 639.1-1143.7 & 905.0 & 675.9-1201.3 & 4.7 & 2549.7 & 1680.9-3632.6 & 2727.1 & 1821.3-3836.0 & 7.0 \\ 85-89 & 1926.8 & 1452.3-2461.8 & 1910.2 & 1485.8-2414.0 & -0.9 & 3740.5 & 2501.7-5147.6 & 3764.3 & 2533.0-5193.4 & 0.6 \\ 90-94 & 4220.0 & 3269.6-5201.0 & 3990.3 & 3122.5-4909.5 & -5.4 & 4841.9 & 3217.3-6643.3 & 4577.3 & 3065.3-6261.8 & -5.5 \\ 95+ & 6305.6 & 4862.3-8162.3 & 5817.5 & 4544.8-7437.8 & -7.7 & 5818.0 & 3781.3-8254.4 & 5320.7 & 3485.5-7465.1 & -8.5 \\ \text { Goiás } & & & & & & & & & & \\ 60-64 & 20.2 & 14.3-28.8 & 20.1 & 14.3-28.5 & -0.2 & 152.6 & 94.9-231.5 & 150.8 & 95.1-225.0 & -1.2 \\ 65-69 & 53.0 & 38.3-72.1 & 56.9 & 40.0-79.3 & 7.2 & 318.2 & 202.7-472.5 & 336.7 & 213.6-505.4 & 5.8 \\ 70-74 & 144.0 & 102.1-198.2 & 160.3 & 111.7-220.3 & 11.3 & 723.8 & 465.8-1032.0 & 806.8 & 509.5-1163.4 & 11.5 \\ 75-79 & 366.4 & 266.5-484.1 & 409.6 & 296.6-546.7 & 11.8 & 1288.0 & 849.3-1836.8 & 1435.0 & 937.1-2073.1 & 11.4 \\ 80-84 & 837.1 & 630.8-1101.1 & 899.8 & 674.2-1195.0 & 7.5 & 2520.1 & 1672.0-3557.6 & 2699.0 & 1779.7-3828.6 & 7.1 \\ 85-89 & 1894.9 & 1439.3-2425.2 & 1888.2 & 1432.2-2362.1 & -0.4 & 3710.0 & 2471.7-5132.1 & 3720.9 & 2491.6-5132.3 & 0.3 \\ 90-94 & 4170.2 & 3192.0-5252.1 & 3923.9 & 3077.0-4800.1 & -5.9 & 4823.4 & 3186.0-6565.2 & 4519.8 & 2983.7-6092.0 & -6.3 \\ 95+ & 6384.3 & 4866.7-8265.8 & 5751.1 & 4421.8-7422.3 & -9.9 & 5815.3 & 3744.6-8244.5 & 5246.5 & 3387.2-7428.5 & -9.8\end{array}$

\section{North region}

Pará

\begin{tabular}{ccccccccccc}
$60-64$ & 19.7 & $14.3-27.6$ & 19.8 & $13.9-27.6$ & 0.5 & 151.5 & $95.0-226.5$ & 148.8 & $91.9-221.3$ & -1.7 \\
$65-69$ & 51.5 & $37.4-70.4$ & 55.6 & $38.9-76.2$ & 7.9 & 314.7 & $201.4-476.0$ & 334.9 & $208.7-501.8$ & 6.4 \\
$70-74$ & 140.1 & $99.7-193.3$ & 157.5 & $110.9-217.6$ & 12.4 & 719.7 & $466.7-1017.4$ & 803.5 & $521.1-1134.7$ & 11.6 \\
$75-79$ & 355.9 & $263.6-471.3$ & 399.5 & $290.3-544.2$ & 12.2 & 1280.3 & $836.1-1834.9$ & 1427.8 & $936.7-2041.8$ & 11.5 \\
$80-84$ & 811.7 & $617.2-1073.8$ & 880.5 & $652.8-1173.3$ & 8.5 & 2520.1 & $1665.4-3630.8$ & 2696.4 & $1759.0-3826.6$ & 7.0 \\
$85-89$ & 1817.8 & $1400.1-2293.5$ & 1830.2 & $1383.3-2335.2$ & 0.7 & 3707.8 & $2488.5-5122.7$ & 3736.6 & $2475.4-5208.8$ & 0.8 \\
$90-94$ & 3912.0 & $3131.3-4730.8$ & 3760.4 & $2915.0-4545.0$ & -3.9 & 4831.0 & $3193.3-6625.5$ & 4567.7 & $3035.3-6245.1$ & -5.5 \\
$95+$ & 5983.7 & $4701.4-7677.2$ & 5528.5 & $4315.8-7018.5$ & -7.6 & 5845.5 & $3773.0-8248.3$ & 5330.4 & $3440.7-7572.1$ & -8.8 \\
Rondônia & & & & & & & & & & \\
60-64 & 21.5 & $15.3-30.4$ & 21.2 & $14.9-29.7$ & -1.5 & 153.1 & $94.5-227.6$ & 151.6 & $97.9-224.1$ & -1.0 \\
$65-69$ & 56.6 & $40.2-76.7$ & 58.9 & $40.9-80.7$ & 4.0 & 320.1 & $199.6-477.6$ & 339.2 & $213.7-511.8$ & 6.0 \\
$70-74$ & 151.3 & $106.5-204.7$ & 165.4 & $116.9-231.6$ & 9.3 & 730.3 & $468.2-1048.3$ & 816.4 & $524.4-1188.3$ & 11.8 \\
$75-79$ & 389.7 & $286.9-517.4$ & 424.7 & $309.6-569.5$ & 9.0 & 1293.5 & $838.4-1846.3$ & 1449.0 & $948.0-2071.3$ & 12.0 \\
$80-84$ & 872.3 & $658.5-1160.0$ & 940.4 & $701.5-1237.7$ & 7.8 & 2527.9 & $1666.8-3588.4$ & 2724.3 & $1794.9-3847.7$ & 7.8 \\
$85-89$ & 1973.7 & $1491.2-2528.7$ & 1966.6 & $1494.5-2488.4$ & -0.4 & 3722.1 & $2487.4-5141.2$ & 3762.1 & $2507.8-5202.8$ & 1.1 \\
$90-94$ & 4352.5 & $3357.3-5495.0$ & 4013.8 & $3174.7-4877.2$ & -7.8 & 4854.4 & $3218.7-6562.2$ & 4592.3 & $3029.3-6285.1$ & -5.4 \\
$95+$ & 6620.5 & $5086.4-8597.1$ & 5958.2 & $4663.9-7552.1$ & -10.0 & 5809.7 & $3778.8-8162.4$ & 5346.7 & $3487.6-7621.2$ & -8.0 \\
\hline
\end{tabular}


Table 2. Continuation.

\begin{tabular}{|c|c|c|c|c|c|c|c|c|c|c|}
\hline \multirow{2}{*}{$\begin{array}{l}\text { Age in years } \\
\text { by location } \\
\text { Brazil }\end{array}$} & \multicolumn{5}{|c|}{$\begin{array}{c}\text { Mortality rates } \\
\text { (per 100,000 inhabitants) }\end{array}$} & \multicolumn{5}{|c|}{$\begin{array}{c}\text { YLDs rates } \\
\text { (per 100,000 inhabitants) }\end{array}$} \\
\hline & 2000 & $95 \%$ UI & 2016 & $95 \% \mathrm{UI}$ & $\Delta \%$ & 2000 & $95 \%$ UI & 2016 & $95 \%$ UI & $\Delta \%$ \\
\hline \multicolumn{11}{|c|}{ Northeast region } \\
\hline \multicolumn{11}{|l|}{ Paraíba } \\
\hline $60-64$ & 20.4 & $14.3-28.5$ & 20.1 & $14.0-28.2$ & -1.7 & 153.0 & $97.0-234.4$ & 151.8 & $95.1-228.3$ & -0.8 \\
\hline $65-69$ & 54.2 & $39.0-73.5$ & 56.6 & $39.7-78.0$ & 4.4 & 319.2 & $202.3-480.7$ & 340.0 & $212.3-508.5$ & 6.5 \\
\hline $70-74$ & 144.3 & $102.8-200.9$ & 158.7 & $112.6-218.1$ & 10.0 & 729.5 & 474.3-1035.6 & 814.1 & $519.8-1168.4$ & 11.6 \\
\hline $75-79$ & 369.8 & $270.9-493.9$ & 404.3 & $298.8-542.6$ & 9.3 & 1297.8 & $845.4-1862.1$ & 1449.6 & $946.7-2074.4$ & 11.7 \\
\hline $80-84$ & 852.9 & $639.1-1120.7$ & 897.0 & $667.4-1181.2$ & 5.2 & 2544.8 & $1669.6-3624.6$ & 2730.6 & $1791.7-3903.4$ & 7.3 \\
\hline $85-89$ & 1895.0 & $1458.3-2379.5$ & 1874.4 & $1433.2-2412.1$ & -1.1 & 3736.5 & $2502.1-5156.4$ & 3774.1 & $2542.6-5248.7$ & 1.0 \\
\hline $90-94$ & 4091.3 & $3212.6-4952.9$ & 3826.1 & $3031.0-4656.9$ & -6.5 & 4846.1 & $3217.4-6526.6$ & 4608.4 & $3056.3-6226.8$ & -4.9 \\
\hline $95+$ & 6239.1 & 4828.7-7969.4 & 5568.5 & 4286.8-7167.4 & -10.7 & 5855.3 & $3791.5-8275.6$ & 5355.8 & $3402.3-7624.0$ & -8.5 \\
\hline \multicolumn{11}{|l|}{ Bahia } \\
\hline $60-64$ & 19.6 & $13.9-27.5$ & 19.7 & $13.6-27.8$ & 0.4 & 150.5 & $96.4-226.6$ & 148.9 & $95.3-225.3$ & -1.1 \\
\hline $65-69$ & 51.9 & $37.3-71.5$ & 55.2 & $38.5-74.7$ & 6.4 & 314.9 & $201.1-467.0$ & 334.0 & $211.7-492.4$ & 6.0 \\
\hline $70-74$ & 139.6 & $99.9-192.8$ & 155.3 & $112.0-217.4$ & 11.3 & 721.0 & $462.3-1027.0$ & 802.1 & $517.5-1142.5$ & 11.3 \\
\hline $75-79$ & 358.2 & $264.1-471.5$ & 394.0 & $283.4-527.2$ & 10.0 & 1279.9 & 834.7-1811.1 & 1425.6 & $921.2-2059.7$ & 11.4 \\
\hline $80-84$ & 814.9 & $616.3-1078.8$ & 857.6 & $642.5-1120.3$ & 5.2 & 2514.0 & $1676.0-3545.4$ & 2687.4 & $1784.1-3814.6$ & 6.9 \\
\hline $85-89$ & 1815.5 & $1401.7-2267.8$ & 1787.3 & $1367.6-2273.8$ & -1.6 & 3706.0 & $2451.2-5155.8$ & 3722.7 & $2508.7-5098.5$ & 0.5 \\
\hline $90-94$ & 3943.4 & $3077.8-4839.3$ & 3688.0 & $2942.2-4486.6$ & -6.5 & 4826.0 & $3212.8-6558.5$ & 4549.8 & $3014.8-6211.7$ & -5.7 \\
\hline $95+$ & 5975.3 & $4674.1-7535.9$ & 5401.9 & $4225.3-6821.3$ & -9.6 & 5823.3 & $3771.4-8216.1$ & 5299.7 & $3423.6-7510.4$ & -9.0 \\
\hline \multicolumn{11}{|c|}{ South region } \\
\hline \multicolumn{11}{|c|}{ Rio Grande do Sul } \\
\hline $60-64$ & 20.4 & $14.5-28.2$ & 20.3 & $14.1-28.8$ & -0.6 & 155.9 & $100.5-233.4$ & 155.5 & $99.4-230.2$ & -0.3 \\
\hline $65-69$ & 53.6 & $38.4-73.9$ & 57.6 & $40.6-81.0$ & 7.4 & 327.3 & $209.4-486.7$ & 347.6 & $223.5-516.3$ & 6.2 \\
\hline $70-74$ & 145.7 & 102.7-200.8 & 163.9 & $116.5-223.9$ & 12.5 & 746.6 & $480.4-1066.1$ & 834.7 & $539.6-1183.9$ & 11.8 \\
\hline $75-79$ & 374.4 & $270.2-503.2$ & 413.1 & $303.1-550.2$ & 10.3 & 1328.0 & $866.9-1894.2$ & 1482.5 & $955.3-2094.1$ & 11.6 \\
\hline $80-84$ & 857.1 & $646.2-1132.6$ & 904.6 & $681.6-1188.3$ & 5.5 & 2606.9 & $1691.7-3727.1$ & 2799.9 & $1867.7-4002.8$ & 7.4 \\
\hline $85-89$ & 1922.1 & $1459.1-2465.9$ & 1886.8 & $1423.1-2406.6$ & -1.8 & 3837.1 & $2573.4-5262.7$ & 3865.6 & $2623.1-5379.3$ & 0.7 \\
\hline $90-94$ & 4166.0 & $3203.3-5220.7$ & 3885.0 & $3088.4-4738.8$ & -6.7 & 4974.6 & $3293.1-6778.5$ & 4724.5 & $3193.0-6406.0$ & -5.0 \\
\hline $95+$ & 6319.0 & $4743.8-8390.1$ & 5699.8 & 4473.3-7286.4 & -9.8 & 5957.4 & $3804.4-8481.2$ & 5453.4 & $3567.4-7714.5$ & -8.5 \\
\hline \multicolumn{11}{|l|}{ Paraná } \\
\hline $60-64$ & 20.5 & $14.3-28.8$ & 20.2 & $14.1-28.7$ & -1.3 & 154.7 & $97.2-230.7$ & 153.1 & $98.1-231.7$ & -1.0 \\
\hline $65-69$ & 54.0 & $38.3-74.6$ & 57.1 & $40.2-78.0$ & 5.7 & 323.2 & $201.4-481.4$ & 343.0 & $222.3-514.5$ & 6.1 \\
\hline $70-74$ & 146.2 & $104.5-202.5$ & 162.1 & $113.9-223.4$ & 10.9 & 736.2 & $476.2-1041.9$ & 820.5 & $526.3-1182.7$ & 11.5 \\
\hline $75-79$ & 376.8 & $273.1-498.2$ & 411.7 & $298.8-547.4$ & 9.3 & 1309.4 & $855.6-1880.2$ & 1458.4 & $944.1-2072.0$ & 11.4 \\
\hline $80-84$ & 858.8 & $646.3-1136.1$ & 900.1 & $682.4-1194.7$ & 4.8 & 2567.5 & $1688.5-3650.8$ & 2747.2 & 1799.3-3903.6 & 7.0 \\
\hline $85-89$ & 1923.3 & $1465.5-2490.1$ & 1893.1 & $1458.2-2365.4$ & -1.6 & 3774.8 & $2496.9-5220.5$ & 3793.3 & $2573.4-5231.4$ & 0.5 \\
\hline $90-94$ & 4182.7 & $3208.6-5217.3$ & 3924.6 & $3125.0-4761.9$ & -6.2 & 4891.3 & $3280.3-6592.3$ & 4613.2 & $3056.3-6258.3$ & -5.7 \\
\hline $95+$ & 6355.5 & $4752.3-8355.6$ & 5748.7 & $4421.2-7372.9$ & -9.5 & 5864.4 & $3824.5-8338.3$ & 5339.9 & $3455.7-7629.4$ & -8.9 \\
\hline \multicolumn{11}{|c|}{ Southeast region } \\
\hline \multicolumn{11}{|l|}{ São Paulo } \\
\hline 60-64 & 23.3 & $17.1-31.6$ & 23.6 & $16.8-33.5$ & 1.2 & 178.0 & $117.9-259.0$ & 181.0 & $116.8-268.8$ & 1.7 \\
\hline $65-69$ & 57.6 & $43.6-74.3$ & 68.0 & $49.8-92.8$ & 18.0 & 344.5 & $233.3-498.9$ & 413.1 & $269.4-616.2$ & 19.9 \\
\hline $70-74$ & 146.8 & $112.7-189.7$ & 190.0 & 139.6-252.6 & 29.4 & 742.1 & $508.7-1020.3$ & 981.1 & $638.6-1356.5$ & 32.2 \\
\hline $75-79$ & 374.2 & $288.7-470.5$ & 470.9 & $352.9-604.3$ & 25.8 & 1297.7 & $893.2-1773.6$ & 1694.5 & $1128.6-2401.8$ & 30.6 \\
\hline $80-84$ & 862.9 & $675.4-1098.7$ & 1010.3 & $784.6-1292.8$ & 17.1 & 2555.2 & $1766.2-3433.9$ & 3088.0 & $2099.6-4231.1$ & 20.9 \\
\hline $85-89$ & 1983.3 & $1534.4-2509.2$ & 2071.3 & $1620.9-2560.7$ & 4.4 & 3859.6 & $2672.8-5186.4$ & 4167.0 & $2813.4-5679.1$ & 8.0 \\
\hline $90-94$ & 4503.8 & $3589.7-5597.6$ & 4232.6 & $3389.2-5070.3$ & -6.0 & 5276.3 & $3711.0-7024.9$ & 5043.8 & $3438.7-6779.2$ & -4.4 \\
\hline $95+$ & 6991.0 & $5450.6-8842.7$ & 6175.9 & 4877.7-7705.9 & -11.7 & 6501.1 & $4362.0-8788.0$ & 5778.8 & $3883.9-8027.0$ & -11.1 \\
\hline
\end{tabular}


Table 2. Continuation.

\begin{tabular}{|c|c|c|c|c|c|c|c|c|c|c|}
\hline \multirow{2}{*}{$\begin{array}{l}\text { Age in years } \\
\text { by location } \\
\text { Brazil } \\
\end{array}$} & \multicolumn{5}{|c|}{$\begin{array}{c}\text { Mortality rates } \\
\text { (per } 100,000 \text { inhabitants) }\end{array}$} & \multicolumn{5}{|c|}{$\begin{array}{c}\text { YLDs rates } \\
\text { (per 100,000 inhabitants) }\end{array}$} \\
\hline & 2000 & $95 \% \mathrm{UI}$ & 2016 & $95 \%$ UI & $\Delta \%$ & 2000 & $95 \%$ UI & 2016 & $95 \%$ UI & $\Delta \%$ \\
\hline \multicolumn{11}{|c|}{ Rio de Janeiro } \\
\hline $60-64$ & 20.4 & $14.4-28.9$ & 20.1 & $14.1-28.8$ & -1.4 & 152.1 & $96.4-229.7$ & 96.5 & $96.5-225.0$ & -0.2 \\
\hline $65-69$ & 54.1 & $38.1-73.6$ & 56.9 & 40.0-78.1 & 5.3 & 318.0 & $201.6-476.0$ & 214.0 & $214.0-507.0$ & 7.4 \\
\hline $70-74$ & 146.2 & 104.3-197.2 & 161.3 & $114.5-222.1$ & 10.3 & 727.1 & $470.0-1028.6$ & 533.1 & $533.1-1164.9$ & 12.5 \\
\hline $75-79$ & 378.2 & $275.9-499.1$ & 411.2 & $301.4-548.5$ & 8.7 & 1298.4 & $840.5-1854.4$ & 946.2 & $946.2-2079.5$ & 11.8 \\
\hline $80-84$ & 855.4 & $639.1-1144.4$ & 903.5 & $676.8-1191.2$ & 5.6 & 2556.7 & $1695.6-3647.7$ & 1807.5 & $1807.5-3902.1$ & 7.3 \\
\hline $85-89$ & 1934.6 & $1462.3-2467.0$ & 1897.1 & $1461.8-2419.6$ & -1.9 & 3776.6 & $2513.3-5248.8$ & 2566.4 & $2566.4-5231.1$ & 0.7 \\
\hline $90-94$ & 4206.5 & $3195.3-5295.2$ & 3945.7 & $3118.7-4823.5$ & -6.2 & 4888.6 & $\begin{array}{c}3250.8- \\
6733.8\end{array}$ & 3039.1 & $3039.1-6306.0$ & -5.1 \\
\hline $95+$ & 6364.6 & $4828.5-8234.8$ & 5761.9 & $4459.6-7346.2$ & -9.5 & 5870.6 & $3791.3-8273.6$ & 3457.6 & $3457.6-7621.8$ & -8.6 \\
\hline
\end{tabular}

YLDs: years lived with disability; 95\%UI: 95\% uncertainty interval; $\Delta \%$ : annual percentage change.

lower than the global dementia cost per capita (approximately $\$ 5,284)^{24}$. Most of these costs are indirect, as the social costs associated with the loss of caregiver productivity, aside from the time and stress experienced by them, are usually informa ${ }^{24}$. Besides patient suffering, the lack of specific coping strategies, whether in primary or specialized care, leads to increased family demand; family members or relatives become informal and untrained caregivers. These caregivers face many challenges, such as diagnosis acceptance, family conflict management, future reprogramming, and, most importantly, engagement in all activities that involve caring for the patient, from providing medications to securing financial and legal support. The overload experienced by caregivers, largely young women, is undoubtedly an indirect cost that can be prevented if taken over by trained professional caregivers ${ }^{25}$.

In this study, although women presented a greater burden of disease, men experienced a greater increase in the burden of $\mathrm{AD}$ and other dementias in the period. Most Brazilian studies showed a higher prevalence of dementia in women, even though the difference was not statistically significant ${ }^{8,9,13}$. The current best explanation for this difference is that women outnumbered men; however, future studies are necessary to clarify this scenario ${ }^{26}$.

The analysis of estimates from the GBD study has certain advantages. Its standardized methodology allows comparisons over time and across places. In addition, UIs reflect the amount and quality of health information for each location and period.

However, one important limitation concerns the paucity of population-based studies on dementia, not only in Brazil but worldwide. We underline that São Paulo presented rates two or three times higher than those of other states, clearly affecting the national average. This finding may be responsible for the great homogeneity of metrics between the states, which prevented a more careful analysis by region. The diversity of diagnostic methods used for dementia is the second limitation. Across the 237 data sources used by the estimates of the GBD-2016 study, 230 diagnostic procedures were used ${ }^{19}$.

The third limitation relates to the impossibility of classifying dementia into subtypes, as different causes of dementia have different clinical and epidemiological profiles accompanied by different prevention and treatment strategies ${ }^{19}$. The primary analysis showed sharp discrepancies between the prevalence of dementia and the cause-of-death data on the specific cause of dementia ${ }^{19}$. In the future, further discrimination between $\mathrm{AD}$, vascular dementia, and other dementia types may be advisable. Doctors are advised not to report dementia as an underlying cause of death on death certificates because this practice can lead to underreporting of deaths, which is difficult to correct ${ }^{27}$. We also highlight the challenge of measuring disability weights since brief descriptions of health status may not fully capture the complexity of diseases such as dementia and $\mathrm{AD}^{19}$.

In conclusion, this study calls attention to the increase in the burden imposed by $\mathrm{AD}$ and other dementias in Brazil, along with the paucity of studies in the country. This reality requires health facilities to invest in prevention and research to ensure a better quality of life not only for those with dementia but also for their families.

\section{ACKNOWLEDGMENTS}

We would like to thank Editage (www.editage.com) for English language editing. 


\section{References}

1. Prince M, Bryce R, Albanese E, Wimo A, Ribeiro W, Ferri CP. The global prevalence of dementia: a systematic review and metaanalysis. Alzheimers Dement. 2013 Jan;9(1):63-75. https://doi.org/10.1016/j. jalz.2012.11.007

2. Etters L, Goodall D, Harrison BE. Caregiver burden among dementia patient caregivers: A review of the literature. J Am Acad Nurse Pract. 2008 Aug;20(8):423-8. https://doi.org/10.1111/j.17457599.2008.00342.x

3. Baumgart M, Snyder HM, Carrillo MC, Fazio S, Kim H, Johns H. Summary of the evidence on modifiable risk factors for cognitive decline and dementia: A population-based perspective. Alzheimers Dement. 2015 Jun;11(6):718-26. https://doi.org/10.1016/j. jalz.2015.05.016

4. GBD 2016 Brazil Collaborators. Burden of disease in Brazil, 19902016. A systematic analysis for the Global Burden of Disease 2016. Lancet. 2018 Sep;392(10149):760-75. https://doi.org/10.1016/ S0140-6736(18)31221-2

5. Murray CJ, Ezzati M, Flaxman AD, Lim S, Lozano R, Michaud C, et al. GBD 2010: a multi-investigator collaboration for global comparative descriptive epidemiology. Lancet. 2012 Dec:380(9859):2055-8. https://doi.org/10.1016/s0140-6736(12)62134-5

6. Murray CJ, Ezzati M, Flaxman AD, Lim S, Lozano R, Michaud C, et al. GBD 2010: design, definitions, and metrics. Lancet. 2012 Dec;380(9859):2055-8. https://doi.org/10.1016/s01406736(12)61899-6

7. Nitrini R, Bottino CM, Albala C, Custodio Capanay NS, Ketzoian C, Llibre Rodriguez JJ, et al. Prevalence of dementia in Latin America: a collaborative study of population-based cohorts. Int Psychogeriatr. 2009 Aug;21(4):622-30. https://doi.org/10.1017/ s1041610209009430

8. Herrera E Jr, Caramelli P, Silveira AS, Nitrini R. Epidemiologic survey of dementia in a community-dwelling Brazilian population. Alzheimer Dis Assoc Disord. 2002 Apr-Jun;16(2):103-8. https://doi.org/10.1097/00002093-200204000-00007

9. Lopes MA, Ferrioli E, Nakano EY, Litvoc J, Bottino CM. High prevalence of dementia in a community-based survey of older people from Brazil: Association with intellectual activity rather than education. J Alzheimers Dis. 2012 Oct;32(2):307-16. https://doi. org/10.3233/jad-2012-120847

10. Bottino CM, Azevedo D Jr, Tatsch M, Hototian SR, Moscoso MA, Folquitto J, et al. Estimate of dementia prevalence in a community sample from São Paulo, Brazil. Dement Geriatr Cogn Disord. 2008 Oct;26(4):291-9. https://doi.org/10.1159/000161053

11. Cesar KG, Brucki SM, Takada LT, Nascimento LF, Gomes $\mathrm{CM}$, Almeida MC, et al. Prevalence of cognitive impairment without dementia and dementia in Tremembé, Brazil. Alzheimer Dis Assoc Disord. 2016 Jul-Sep;30(3):264-71. https://doi.org/10.1097/ wad. 0000000000000122

12. Caramelli P, Teixeira AL, Barbosa MT, Santos AP, Pellizzaro M, Guimaraes HC, et al. Prevalence of cognitive impairment and dementia in a cohort of oldest old in Brazil:The Pietà study. Alzheimers Dement. 2009 Jul;5(4):P391-2. https://doi.org/10.1016/j. jalz.2009.04.1037

13. Nitrini R, Caramelli P, Herrera E Jr, Bahia VS, Caixeta LF, Radanovic $\mathrm{M}$, et al. Incidence of dementia in a community-dwelling Brazilian population. Alzheimer Dis Assoc Disord. 2004 Oct-Dec;18(4):241-6.
14. Meguro K, Chubaci RYS, Meguro M, Kawamorida K, Nobuko G, Caramelli P. Incidence of dementia and cause of death in elderly Japanese emigrants to Brazil before World War II. Arch Gerontol Geriatr. 2011 JanFeb;52(1):75-8. https://doi.org/10.1016/j.archger.2010.02.003

15. Nitrini R, Caramelli P, Herrera E Jr, de Castro I, Bahia VS, Anghinah $\mathrm{R}$, et al. Mortality from dementia in a community-dwelling Brazilian population. Int J Geriatr Psychiatry. 2005 Mar;20(3):247-53. https:// doi.org/10.1002/gps.1274

16. Chaves ML, Camozzato AL, Godinho C, Piazenski I, Kaye J. Incidence of mild cognitive impairment and Alzheimer disease in Southern Brazil J Geriatr Psychiatry Neurol. 2009 Mar;22(3):181-7. https://doi. org/10.1177/0891988709332942

17. Souza MFM, França EB, Cavalcante A. Burden of disease and health situation analysis: results of the Global Burden of Disease (GBD) Brazil network. Rev Bras Epidemiol. 2017 May;20(Suppl 1):1-3. http:// dx.doi.org/10.1590/1980-5497201700050001

18. Brasil. Instituto Brasileiro de Geografia e Estatística. Censo 2010. Available from: https://censo2010.ibge.gov.br

19. GBD 2016 Dementia Collaborators. Global, regional, and national burden of Alzheimer's disease and other dementias, 1990-2016: a systematic analysis for the Global Burden of Disease Study 2016. Lancet Neurol. 2019 Jan;18(1):88-106. https://doi.org/10.1097/01. ogx.0000511935.64476.66

20. Foreman K, Lozano R, Lopez AD, Murray CJ. Modeling causes of death. An integrated approach using CODEm. Popul Health Metrics. 2012 Jan;10:1. https://doi.org/10.1186/1478-7954-10-1

21. Naghavi M, Abajobir AA, Abbafati C, Abbas KM, Abd-Allah F, Abera SF, et al. Global, regional, and national age-sex specific mortality for 264 causes of death, 1980-2016: a systematic analysis for the Global Burden of Disease Study 2016. Lancet. 2017 Sep;390(10100):115110. https://doi.org/10.1016/S0140-6736(17)32152-9

22. Beltrão KI, Camarano AA, Kanso S. Dinâmica populacional brasileira na virada do século XX. Rio de Janeiro: IPEA; 2004 (Texto para Discussão, 1.034). Available from: http://www.ipea.gov.br/agencia/ images/stories/PDFs/TDs/td_1034.pdf

23. Vos T, Abajobir AA, Kalkidan HA, Abbafati C, Abbas KM, Abd-Allah F, et al. Global, regional, and national incidence, prevalence, and years lived with disability for 328 diseases and injuries for 195 countries, 1990-2016: a systematic analysis for the Global Burden of Disease Study 2016. Lancet. 2017 Sep;390(10100):1211-59. https://doi.org/1 $0.3410 / f .731220250 .793569875$

24. Ferretti C, Sarti FM, Nitrini R, Ferreira FF, Brucki SM. An assessment of direct and indirect costs of dementia in Brazil. PLoS ONE. 2018 Mar;13(3):e0193209. https://doi.org/10.1371/journal.pone.0193209

25. Silva CF, Passos VMA, Barreto SM. Frequência e repercussão da sobrecarga de cuidadoras familiares de idosos com demência. Rev Bras Geriatr Gerontol. 2012 Oct-Dec;15(4):707-31. https://doi. org/10.1590/s1809-98232012000400011

26. Burlá C, Camarano AA, Kanso S, Fernandes D, Nunes R. Panorama prospectivo das demências no Brasil: um enfoque demográfico. Ciênc Saúde Coletiva. 2013 Oct;18(10):2949-56. https://doi. org/10.1590/s1413-81232013001000019

27. Romero JP, Benito-Leona J, Mitchell AJ, Trincado R, Bermejo-Pareja F Under reporting of dementia deaths on death certificates using data from a population-based study (NEDICES). J Alzheimers Dis. 2014 Feb;39(4):741-8. https://doi.org/10.3233/jad-131622 